\title{
On the Challenges and Opportunities of Smart Meters in Smart Homes and Smart Grids
}

\author{
Zainab Al-Waisi, Michael Opoku Agyeman \\ Department of Computer and Immersive Technologies \\ University of Northampton, Email: Michael.OpokuAgyeman@northampton.ac.uk
}

\begin{abstract}
Nowadays, electricity companies have started applying smart grid in their systems rather than the conventional electrical grid (manual grid). Smart grid produces an efficient and effective energy management and control, reduces the cost of production, saves energy and it is more reliable compared to the conventional grid. As an advanced energy meter, smart meters can measure the power consumption as well as monitor and control electrical devices. Smart meters have been adopted in many countries since the 2000 s as they provide economic, social and environmental benefits for multiple stakeholders. The design of smart meter can be customized depending on the customer and the utility company needs. There are different sensors and devices supported by dedicated communication infrastructure which can be utilized to implement smart meters. This paper presents a study of the challenges associated with smart meters, smart homes and smart grids as an effort to highlight opportunities for emerging research and industrial solutions.
\end{abstract}

\section{INTRODUCTION}

Traditionally, electromechanical meters or basic electronic meters have been used to measure energy consumption [1]. These types of meters require sending the suppliers to the energy meter location for meter readings and other management tasks such as meter disconnection. This is exercabating considering the high number of customers [2]. Smart meters have become increasingly popular for calculating, controlling and measuring power consumption, gas and water (Figure 1). A smart meter is an electronic device which used to record and transmit the information of electricity, water or gas consumption. The data is then normally stored on a server which will be used for further operations like calculating the consumption fees, showing consumption statistics or showing other information to the customer [3], [4]. It is expected that based on smart meter information, significant energy and financial savings can be achieved [5]. The large-scale installations of smart meters will generate a massive amount of data which can offer the company a unique insight of the power consumption of different consumers. This information can be used to help consumers to shift their consumption from peak hours, which can result in significant savings of the energy [6]. Also, it can help electricity scheduling to facilitate safe and efficient operation of the power system. Smart cities adopt smart grid and smart meters.

Figure 2 and Table I shows the difference between smart meters and conventional meters as well as smart grids and conventional grids, respectively. As shown in Figure 2 smart meters provide efficient communication between customers and utility companies. By using smart meters, the system will automatically send the information from the client unit to the central unit and vice versa. The client unit is responsible for

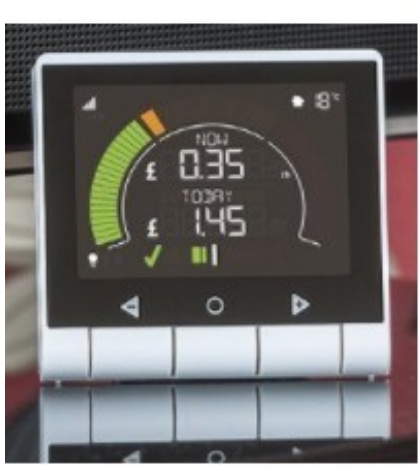

Smart Electricity Meter

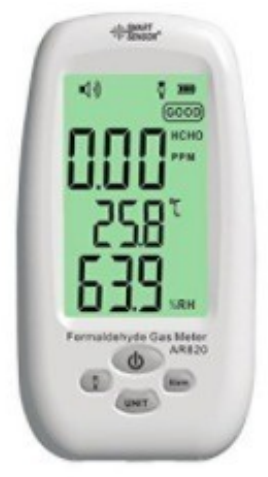

Smart GAS Meter
Fig. 1. An example model of smart eletric meter and smart gas meter

Conventional Meters

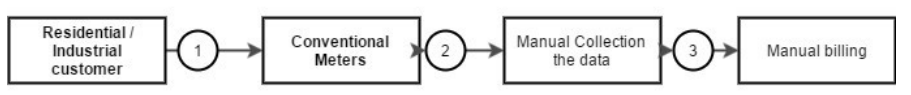

Smart Meters

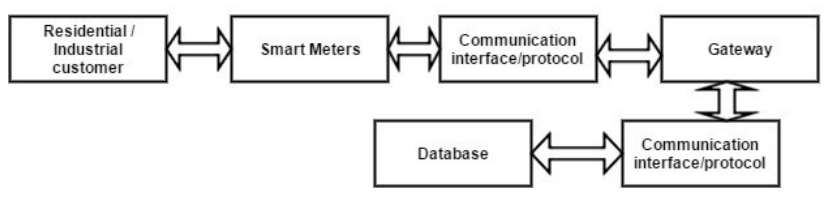

Fig. 2. Metering architecture (Conventional Meter Vs Smart Meter) [7]

TABLE I. COMPARISON BETWEEN

SMART GRID AND CONVENTIONAL GRID

\begin{tabular}{|l|l|}
\hline Conventional Grid & Smart Grid \\
\hline $\begin{array}{l}\text { Electromechanical } \\
\text { meters }\end{array}$ & Digital Meters \\
\hline $\begin{array}{l}\text { One way } \\
\text { communication }\end{array}$ & Two-way communications \\
\hline Few sensors & Multiple sensors \\
\hline Manual monitoring & Automatic monitoring \\
\hline Limited control & Unlimited control \\
\hline $\begin{array}{l}\text { Few Customer } \\
\text { choices }\end{array}$ & Many customer choices \\
\hline $\begin{array}{l}\text { Manually generate } \\
\text { fees }\end{array}$ & Automatically generate fees \\
\hline $\begin{array}{l}\text { Centralized } \\
\text { generation }\end{array}$ & Distribution generation \\
\hline
\end{tabular}

distributing data over the network to the utility company. Thus, the utility company has the ability to produce a billing for the customer, which has all information about the energy consumption and other information. Moreover, Table I shows that 
smart grid can be equipped to automatically monitor the energy unlike the conventional grid. Smart metering technology allows the ease to switch between suppliers and different payments. In addition to providing the consumers and electricity suppliers with information on the current electricity consumptions, smart meters enhance the monitoring of the quality of the power supply for different connections.

This paper presents a study of recent contribution to smart metering in smart homes and smart grids. Particularly, we explore the challenges associated with smart meter design in order to identify research and design opportunities. The remainder of the paper is structured as follows: Section II, presents a background reading of smart grids, smart homes and smart meter architectures. Section III dicusses various enabling communication technologies for smart meter design. Section IV, discusses the challenges associated with smart meters, smart home and smart grid. Here, various security measures are also discussed. Finally, Section V concludes the paper.

\section{BACKGROUND READING}

\section{A. Home Energy Management System}

The Home Energy Management System (HEMS) is used to manage the power supply of a specific house. The main reason for using HEMS is to reduce the energy consumption by encouraging the consumers to reduce the power consumption when the energy becomes high. Advanced Metering Infrastructure (AMI) is the network between smart grid and the services of the home network, whereas, Automatic Metering Reading (AMR) and Automatic Metering Management (AMM) are implemented using a smart meter. A smart meter is an important component in AMI and support all AMR operations by monitoring and controlling electrical devices, energy consumption at home. The smart meter used to send the data to the utility company for making a decision of generating and distributing the power. In addition, generating the bill to the consumer. The utility company sends the smart meter data to the consumer and display the energy consumption prices on smart meter display (e.g. LCD) in order to encourage the customer to reduce their power consumption as well as have more information the about energy consumption of their electrical devices. The main advantage is that, smart meters give accurate monitoring and control of the power supply. The smart meter requires communication infrastructure and gateway in order to collect all individual smart meter data. Furthermore, smart meters can be used to automatically control smart home electrical devices [8].

\section{B. An Overview of Smart Grid and Smart Home}

1) Smart Grid: The term of smart grid refers to the modern power grid, which involves the generation, distribution, management, controlling, and automatically collection of electricity consumption data [7]. A smart grid is an electrical grid which uses (digital and/or analog signals (modern technology)) in order to collect and communicate the electricity information of both suppliers and consumers. Smart grids installation improve the efficiency of the system by contributing to the efficient transmission and distribution of the electricity. The installation of the smart grid means to change the electricity system infrastructure. For example, the replacement of electromechanical and digital meters with smart meters to promote sustainability and system efficiency [9].

Nowadays, the architecture of smart grid has been described using several frameworks, U.S. National Institute of Standards and Technology (NIST) [10] proposed a model which can be considered as one of the most widely adopted. This model visualizes the smart grid as a set of seven interconnected domains. These domains are (Clients, Markets, Service Providers, Operations, Bulk Generation, Transmission, and Distribution). The first four domains (Transmission, Distributed, Customers, and Bulk Generation) focus on generating and distributing the energy of smart grid. Also, these domains are for ensuring that the communication is effective between the customer side and AMI side. However, the other remaining domains (Markets, Operations, and Service providers) are responsible for managing, distributing market energy.

For this case study, the architecture of smart grid proposed by [11] is adopted. Figure 3 shows the different layers of the smart grid. The first layer represents the power consumers (e.g. homes, buildings or industrial areas, electrical vehicles, etc.). These different types of the power consumers connect to the internet via wire or wireless network, that connect appliances with smart meters and energy management device, responsible for reporting the energy consumption to the grid at any given time as well as sending messages from the grid back to the meter. The first layer is therefore considered as the unit responsible for collecting electricity consumption [11], [12].

The second layer, one can find Neighbourhood Area Networks (NANs) i.e., networks that cover small geographic areas which are responsible for connecting the smart meter with the distribution access point, which used to send the collected data to the third layer. Remote Terminal Device (RTD) is an electronic device used to transmit the data to the Supervisory Control and Data Acquisition System (SCADA) system at (third layer). The second layer would be considered as the sending information unit [10], [12].

At the third layer (top) one can find Wide Area Networks (WANs) used to connect multiple NANs. All the data collected by the NANs is delivered at this top layer. The SCADA is esponsible for managing and distributing the received data. The Meter data Management (MDMS) is responsible for billing the customer depends on their consumption. The Demand Response Management Systems (DRMS), the Lord Management Systems (LMS), the Outage Management Systems (OMS) and the Customer Information Systems (CIS), can all found in this third layer [10], [12].

2) Smart Home architecture : The architecture of smart home consists of two parts (internal, and external environment). The external environment of the smart home consists of smart grid entities and the entity which is responsible for connecting the smart home with the smart grid. On the other hand, internal environment consists of all electrical appliances and devices belonging to the smart home. The entity inside the smart home managing these appliances can be called a smart meter. Specific entities within the network of smart home represent the internal and external environments. An entity called Entity Services Interface (ESI) represents the external environment. However, Energy Management system 


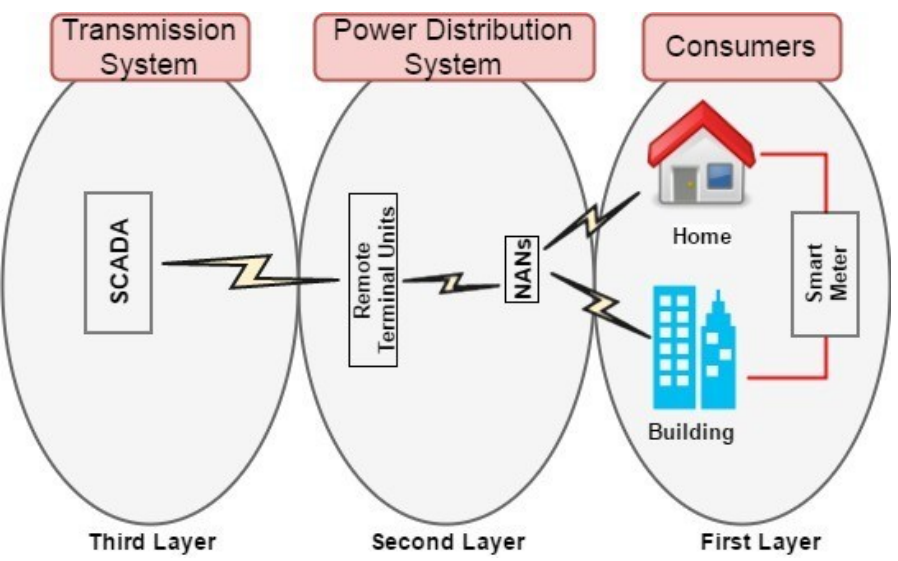

Fig. 3. Smart Grid layers

(EMS), represent the internal environment. The ESI can be considered as the interface between smart home and smart grid. It enables to control of all appliances(e.g. light switches, washing machine, air conditions, etc.) and the distribution of the energy resources to the neighbourhood collection points. Also, it used for monitoring all the data. Thus, ESI responsible for sending the information from internal environment to the external environment and send these data to the control unit via the internet. Figure 4 shows the internal and external environment of smart home, highlighting the EMS and ESI.

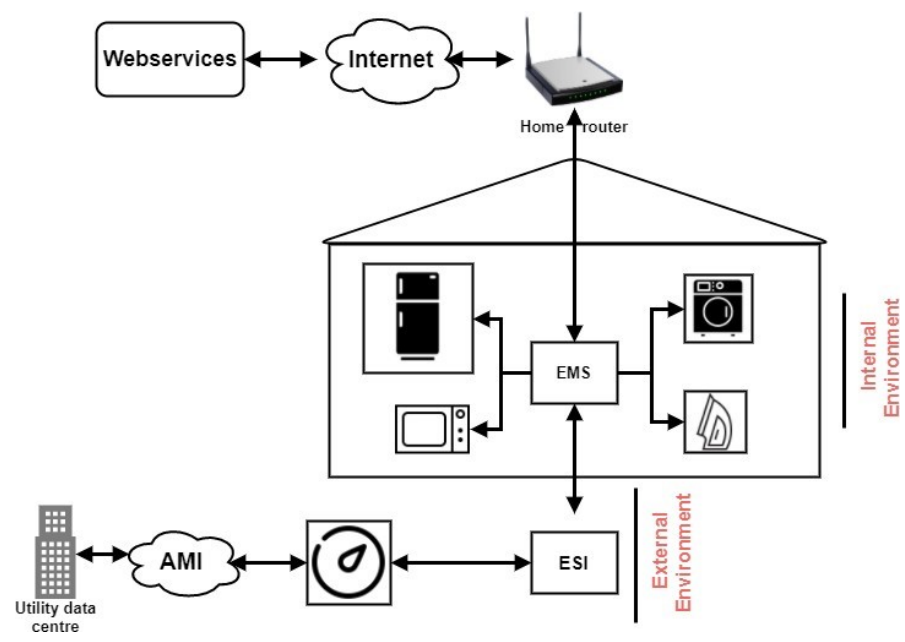

Fig. 4. Smart Home Architecture

The main functionalities of each component in a smart home are:

1) Utility company: responsible for managing, controlling meter data, generating bills and etc. Usually, the utility company connects to the (data-concentrator network) through WAN and the communication channel can be (e.g. WIFI, satellite, etc.).

2) Data-concentrator network: the communication between (data-concentrator network) and smart meter might be through (e.g. Wi-Fi, power line carrier, ZigBee, etc.). Data-concentrator consists of smart meters and the data collector. Usually, the form of a smart meter is a wireless mesh network. The meter reading data will be forwarded to the data collector through different communications (e.g. multi-hop communication). Then the data collector will transmit the data to the utility company.

3) Home Area Network: this network has a gateway which receives the power consumption from the smart meter and displays it on (e.g. LCD display, Smartphone, laptop, etc.). The consumer has the ability to monitor and control the smart meter as well as remotely control the appliances in their home.

4) Smart meter: used to measure the power consumption of any electrical device and send the data to the data collector through Home Area Network. The data is then sent to the utility company.

\section{Why Smart Meters?}

The term smart meter refers to the meter functionalities of remote control of the energy, the automatic measurement of the electricity consumption and the generation of bills. It was called AMR which used one-way communication and the ability to automatically read the electricity usage each month. The capability of AMR was a simple functionally to read the electricity consumption. However over time, a major upgrade of meter functionalities occurred after integrating the meter with two-way communication, which has been called AMI [13]. AMI constitutes monitoring and recording of the energy usage information as well as ease to transfer these data between the utility company and the consumers. Smart meters enable real-time pricin and data for the consumer and utility company; remote control of appliances and operations; monitoring of power quality; easy detection of energy theft; communication with all other intelligent devices at home; easy to transfer meters' information from home to the utility company throughout the network; easy to notify the consumers about the energy consumption; enhanced safety and eco-friendliness [14]. Table 2 shows the issues of conventional meters and what the benefits of replacing them with smart meters.

TABLE II. Benefits of Smart Meters

\begin{tabular}{|l|l|}
\hline Problems & Solutions \\
\hline $\begin{array}{l}\text { Cost: employee } \\
\text { working at a } \\
\text { specific company } \\
\text { to gather smart meter } \\
\text { consumption from } \\
\text { houses. }\end{array}$ & $\begin{array}{l}\text { By using smart meter, there is no need to have an } \\
\text { employe to gather smart meter data. }\end{array}$ \\
\hline $\begin{array}{l}\text { Time: sending the } \\
\text { employee to the } \\
\text { consumers home is } \\
\text { time consuming. }\end{array}$ & $\begin{array}{l}\text { By a short time the data will be sent from smart meter } \\
\text { to the server and vice versa. }\end{array}$ \\
\hline $\begin{array}{l}\text { Security issue: } \\
\text { within the traditional } \\
\text { meters. Information } \\
\text { related to the } \\
\text { consumers can be } \\
\text { lost at any time. }\end{array}$ & $\begin{array}{l}\text { With smart meter all information encrypted and saved in } \\
\text { Difficult to manage } \\
\text { and control smart } \\
\text { meter device. }\end{array}$ \\
\hline $\begin{array}{l}\text { Consume more } \\
\text { energy }\end{array}$ & Easy to manage and control smart meter \\
\hline
\end{tabular}

However, conventional electromechanical meters served as the utility cash register for the most of its history. In other words, these meters used to record the total energy consumption over a period of time. Smart meter design incolves 
the following key components [9]: 1. Smart meter circuit board with the communication module; 2 . Server/control unit; 3. Data concentrator unit; 4. Mobile device/Website/ screen for displaying different information; 5. Another devices and sensors; Generally, the energy consumed measured by the smart meter (using a specific sensor) will be sent to the central unit. After that, the central unit sends this information to the client unit. Figure 5 illustrates the architecture of smart meter working. The key features of a smart meter can be summarised as: a) Two-way communication; b) Automatically collect the data; c) Store the data in a safe place such as (database); d) Automatically generate the bill for consumers; e) Realtime measure the electricity consumptions; f) Display data; g) Security functions

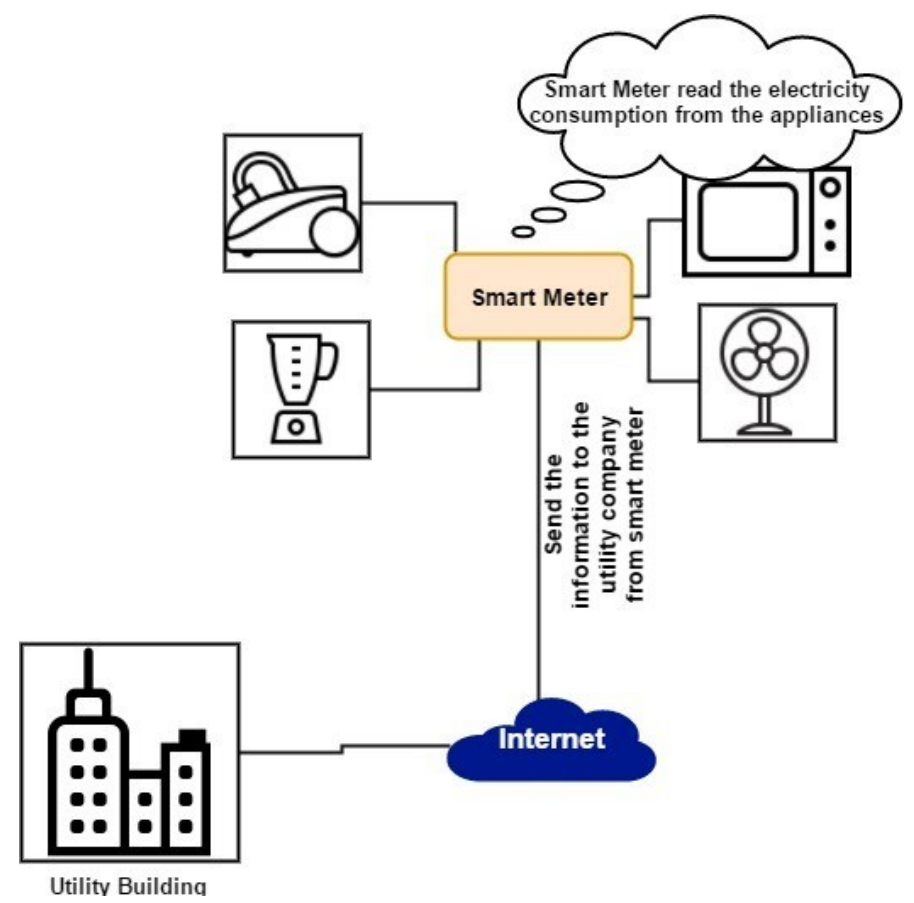

Fig. 5. Smart meter

\section{Smart Meter TeChNOlogies}

Smart meters vary in technology and design. However, all smart meters operate through a simple overall process [15]. Smart meter collect the data from customers appliances and transmit the meter data to data collector through the internet (e.g. Local Area Network (LAN)). The data can be transmitted every 50-60 minutes or once a day. The data collector then sends the meter information to the utility company through WAN.

There are two basics types of meter system communication technologies: (i) Radio Frequency (RF) (ii) Power Line Carrier (PLC). The utility company chooses the best technology depending on their needs. There are different factors which affect the selection of technology, such as [15]: 1. Existing infrastructure. 2. Economic impact to the utilitys customer, as well as impact on legacy equipment, technical requirements, and functionality.

\section{A. Radio Frequency $(R F)$}

With Radio Frequency (RF) communication technology, the smart meter collects the data from the consumer then send it to the data collector through a wireless radio. Afterwards, the data is delivered to the utility. There are two types of RF technologies:

Point-to-Point Technology: Within this technology, the smart meter communicates directly to with the collector, usually a tower. Various techniques have been proposed to transmit the data from the smart meter to the utility company via a tower collector [15]. Point-to-Point have several benefits such as large bandwidth, direct communication with the endpoint and enhanced throughput. However, Point-to-Point RF technology suffers from issues with remote areas (with topography and long distances) and interface with distribution automation devices.

Mesh Technology: With Mesh RF technology, smart meters communicate with each other via a Local Area Networ (LAN) cloud form at the collection point. The data is transmitted to the utility using various Wide Area Network (WAN) methods [16]. Mesh has a wide bandwidth with operation frequency and acceptable latency of about $915 \mathrm{MHz}$. However, Mesh technology has some disadvantages also suffers from remote area coverage.

\section{B. Power Line Carrier (PLC):}

Power Line Carrier (PLC) technology allows data to be transfered between the smart meter and the utility company using the utility power lines. PLCs are cost-effective for rural lines which makes it possible to work with over long distance. On another hand, PLCs have long latencies for data transmission (compared to RF technology), less bandwidth and higher cost in cities.

In the conclusion, the utility company have to choose the most suitable communication technology depending on their needs.

\section{Meter Issues AND CHALlengeS}

In general, replacing the traditional meter with a smart meter can be done with more advantages. However, the design, deployment and maintenance of the smart meter lead to different issues and challenges. Furthermore, the implementation of a smart meter in a distributed system requires spending a tremendous amount of money to invest as well as the the network and related software tools. Consequently, replacing the conventional meters with a smart meter may be a challenging for utility companies and customers. Though several devices are integrated with smart meter system, the full benefit of these devices extent only when all the appliances and devices in the distribution and metering network are included in the communication network [17], [18]. Integrating of these devices becomes more complicated as a huge number of customers start using the smart meter [17].

Additionally, smart meters create potential privacy and security issues as the data and signals are transmitted via a network. Furthermore, the data might also have different information about the customer (e.g. sensitive information). In addition, having information about the appliances such as 
what appliances are in use, appliances IDs, etc. via a network might pose security threats [19]. In order to communicate the data and control signals with the central unit, smart meters have to run these commands of controlling devices from the utility companies. Smart meters operations involve a huge quantity of data to be transferred between smart meters and the server as well as the consumers system. Thus, securing these data and choosing the right network can be a difficult job (to prevent attackers). Moreover, several smart meters communication networks use a low bandwidth, which leads to generating a high traffic and limits in the quantity of data to be transmitted. These problems make the data unsecure. Integrating of these devices will lead to a huge quantity of data transmitted the need to have a memory to store these data. These requirements could lead to increase the overall deployment costs.

There are different issues related to the security vulnerabilities and these issues might be related to (weak authentication, quality the software, weak protocol, weak network, weak error handling, etc.). In spite of these issues, some utility companies pay less attention to the maintenance of their communication networks and these can lead to safety issues. Even if these companies use wired communication, in this case, a physical damage to the cable might also cause an interruption in data transfer [17].

\section{A. The Security Objectives of Smart Home/Smart Grid}

Smart home/grid security is an important role in smart cities. Nowadays, vulnerabilities of the internet have been increased significantly, so it is important to keep all (e.g. sensors data, wireless connections network, Cloud, Database, consumers information) secured over the network. The security goals of smart home/grid are expected to meet the first step of building smart meters for ensuring the consistent of the smart grid. Thus, to ensure a secure smart meters, it must have the following properties:

Confidentiality: to ensure that data can be accessed only by individuals or systems or trusted people in the utility company. Integrity: to ensure that the reliability and consistency of all data will be maintained.

Availability: to ensure that all data, website, network, servers can be accessed only by any authorized entity. Also, the system should make sure to protect against any threats or attacks.

Authenticity: the system has to ensure that all received messages are sent from authorized people.

Authorization: to ensure that the right identification is used for everybody in the system, in order to ensure access control for everybody such as (admin, staff, and users).

\section{B. Security attacks and smart Home/Grid}

There are two main categories of attacks. Usually, these attacks attempt to compromise the security goals, which have been described in Section IV-A. The first category is Passive attacks. This type of attack usually learn from attacking information without affecting the system resources. In other words, in the passive attack, the attacker usually obtain information being transmitted not to modify it but to learn from it (Figure 6).

The second category is Active attack, which effects the system operations or resources. Active attacks can modification

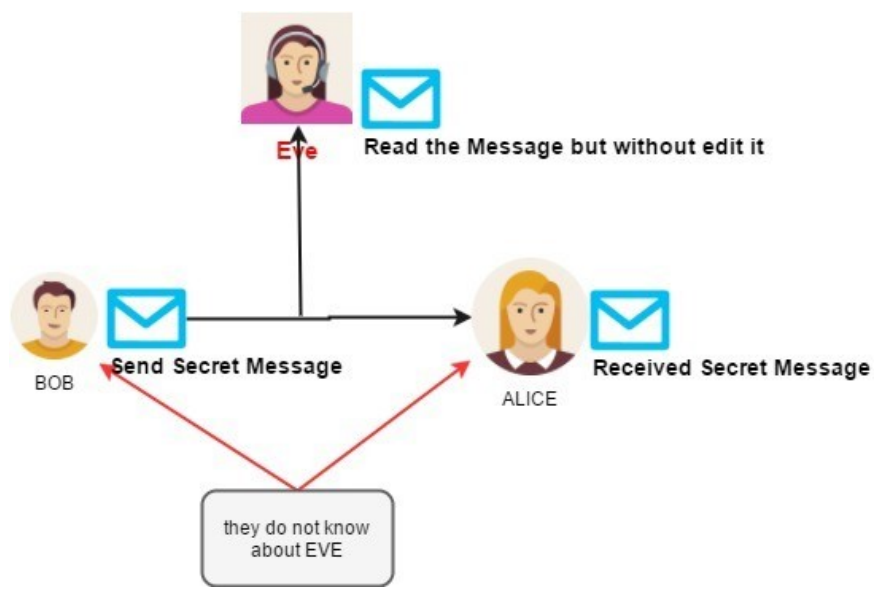

Fig. 6. Passive Attack

the data or cause a negative effect on the system. The most common amongst types of this attack are data modification, denial of service, distributed denial of service, replay, a man in the middle, IP address spoofing, password-based attack, sniffer attack and SQL injection. A data modification attack modifies the original content of the data (message), or reorders the content, or causing a delay with the aim of providing an unauthorized access. A denial of service attack sends different requests (usually about 1000 requests per minute) to the server via the network to interrupt the communication resources of a system. Distributed denial of service is similar to a denial of service attack, but the requests can be sent via multiple compromised resources (usually with more than 1000 requests per minute). A replay attack, capture of a message in a communication and resend it to produce an unauthorized effect. Man in the middle attack usually occurs when the network is in a low level of communication and the data are not encrypted. Thus, a man in the middle attack can easily read the data, control and exchange it or even try to interrupt the communication between two resources. IP address spoofing allows attackers to easily modify or control devices with IP addresses. Password based attacks target the system password so it is important to hash the password to prevent unauthorized access. Sniffer attack tries to view the content of sent and/or receiv data. Thus, if the data is encrypted or hashed successfully, it will be difficult to the attacker to view it or even interpret. Figure 7 shows the process of active attack.

\section{Impact of Federal Information Processing Standard}

Federal Information Processing Standard (FIPS) 199 [20], requires Federal agencies to assess the system information, and they categorize them confidently, integrity, availability, and authenticity into three different categories. These categories are Low (L), Moderate (M), High (H). (i) Low (L): if the violation of any of the security goals can have a limited effect on the operations of smart home/grid, and cause minor damage or minor financial losses on the system; (ii) Moderate (M) if the violation of any of the security goals have a significant effect on the operations of smart home/grid and can cause a significant damage or significant financial loss on the system; (iii) High $(\mathrm{H})$ if the violation of one or more of the security 


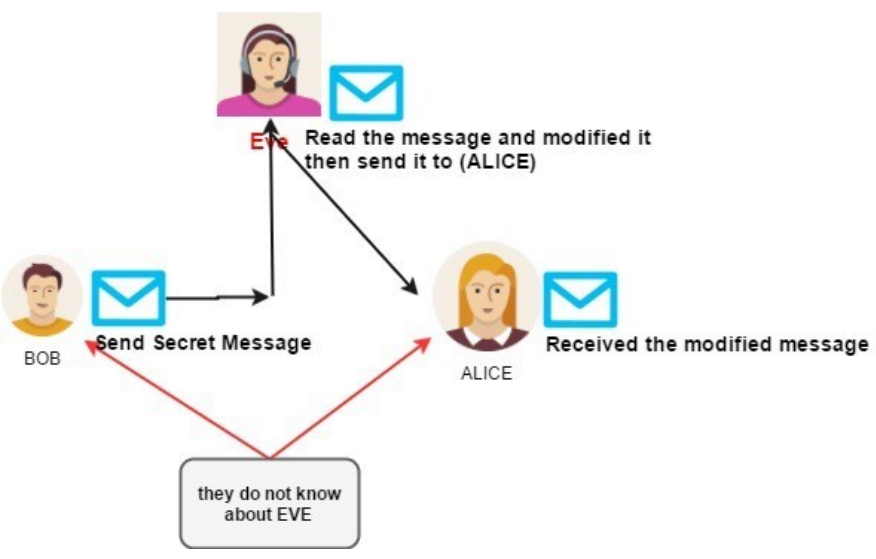

Fig. 7. Active Attack

goals can have a catastrophic effects on smart home/grids operations (this includes a major negative effect on the system or major financial loss). Table III highlights different types of threats and classifies these threats based on FIPS.

TABLE III. Potential Threats of Smart Home/Grid

\begin{tabular}{|l|l|l|}
\hline Threats & Security Goals & FIPS impact \\
\hline Data Modification & Integrity Authenticity & L-M \\
\hline Denial of service & $\begin{array}{l}\text { Integrity Authenticity Availability } \\
\text { Non Repudiation }\end{array}$ & M-H \\
\hline $\begin{array}{l}\text { Distributed denial } \\
\text { of service }\end{array}$ & $\begin{array}{l}\text { Integrity Authenticity Availability } \\
\text { Non Repudiation }\end{array}$ & M-H \\
\hline Replay & Authentication & L-M \\
\hline Man in the Middle & $\begin{array}{l}\text { Integrity Authenticity Availability } \\
\text { Non Repudiation Confidently }\end{array}$ & L-M \\
\hline IP address spoofing & $\begin{array}{l}\text { Confidently Integrity Availability } \\
\text { Confidently }\end{array}$ & L-M \\
\hline $\begin{array}{l}\text { Password based } \\
\text { attack }\end{array}$ & Confidently Integrity Availability & M-H \\
\hline Sniffer attack & Confidently Integrity Availability & M-H \\
\hline SQL injection & $\begin{array}{l}\text { Integrity Authenticity Availability } \\
\text { Non Repudiation Confidently }\end{array}$ & M-H \\
\hline
\end{tabular}

\section{CONCLUSION}

In this paper, we have reviewed several aspects of smart meter. Firstly, a background introduction to smart meter, smart grid and smart homes is presented. Various architectures for designing these technologies are considered. Furthermore, different smart meter communication technologies such as radio frequency, Power Line Carrier are discussed to help designers in making their choise of communication platform as well as encourage future research. Security issues relating to smart metering is explored in details to highlight required security goals, various types of attacks and how these attacks could be avoided or resolved. Future work involves the development of a smart electricity meter that is equipped with both automatic monitoring of energy consumption as well as control of various appliances in a smart home over Internet-of-Things infrastructure.

\section{REFERENCES}

[1] J. Zheng, D. W. Gao, and L. Lin, "Smart meters in smart grid: An overview," in IEEE Green Technologies Conference (GreenTech), April 2013, pp. 57-64.

[2] Z. Jebroni, H. Chadli, B. Tidhaf, A. Benlghazi, and A. Tahani, "Gain correction and phase compensation of a smart electrical energy meter," in International Conference on Engineering MIS (ICEMIS), Sept 2016, pp. 1-6.

[3] S. B. Taieb, R. Huser, R. J. Hyndman, and M. G. Genton, "Forecasting uncertainty in electricity smart meter data by boosting additive quantile regression," IEEE Transactions on Smart Grid, vol. 7, no. 5, pp. 24482455, Sept 2016.

[4] S. Arora and J. W. Taylor, "Forecasting electricity smart meter data using conditional kernel density estimation," Omega, vol. 59, no. Part A, pp. $47-59,2016$, business Analytics.

[5] L. S. N. A. C. VD, "Estimating the impact of time-of-use pricing on irish electricity demand," 2012.

[6] _ "Estimating the impact of time-of-use pricing on irish electricity demand, working paper," 2012.

[7] X. Fan and G. Gong, "Security challenges in smart-grid metering and control systems," vol. 3, pp. 42-49, 072013.

[8] B. Subhash and V. Rajagopal, "Overview of smart metering system in smart grid scenario," in POWER AND ENERGY SYSTEMS: TOWARDS SUSTAINABLE ENERGY, March 2014, pp. 1-6.

[9] S. Patel, U. K. R. Y., and P. K. B., "Role of smart meters in smart city development in india," in IEEE 1st International Conference on Power Electronics, Intelligent Control and Energy Systems (ICPEICES), July 2016, pp. 1-5.

[10] "Nist framework and roadmap for smart grid interoperability standards, release 1.0," 2010, https://www.nist.gov/newsevents/news/2014/10/nist-releases-final-version-smart-grid-frameworkupdate-30.

[11] X. Li, X. Liang, R. Lu, X. Shen, X. Lin, and H. Zhu, "Securing smart grid: cyber attacks, countermeasures, and challenges," IEEE Communications Magazine, vol. 50, no. 8, pp. 38-45, August 2012.

[12] N. Komninos, E. Philippou, and A. Pitsillides, "Survey in smart grid and smart home security: Issues, challenges and countermeasures," IEEE Communications Surveys Tutorials, vol. 16, no. 4, pp. 1933-1954, Fourthquarter 2014.

[13] D. Alahakoon and X. Yu, "Smart electricity meter data intelligence for future energy systems: A survey," IEEE Transactions on Industrial Informatics, vol. 12, no. 1, pp. 425-436, Feb 2016.

[14] R. R. Mohassel, A. Fung, F. Mohammadi, and K. Raahemifar, "A survey on advanced metering infrastructure," International Journal of Electrical Power Energy Systems, vol. 63, no. Supplement C, pp. 473 - 484, 2014.

[15] E. Electrical Institute/EEI and A. MeterComittees, "Smart meters and smart meter systems : A metering industry perspective," p. 29, 012011.

[16] S. Ruj, A. Nayak, and I. Stojmenovic, "A security architecture for data aggregation and access control in smart grids," CoRR, vol. abs/1111.2619, 2011.

[17] S. S. S. R. Depuru, L. Wang, V. Devabhaktuni, and N. Gudi, "Smart meters for power grid , challenges, issues, advantages and status," in IEEE/PES Power Systems Conference and Exposition, March 2011, pp. $1-7$.

[18] "Smart meters, quarterly report to end september," pp. $11-12,2016$, https://www.gov.uk/government/collections/smart-meters-statistics.

[19] C. Bennett and D. Highfill, "Networking ami smart meters," in IEEE Energy 2030 Conference, Nov 2008, pp. 1-8.

[20] P. J. B. A. L. B. J. D. L. Evans, "Standards for security categorization of federal information and information systems," pp. $11-12,2004$. 